\title{
An Efficient Periodic Broadcasting with Small Latency and Buffer Demand for Near Video on Demand
}

\author{
Ying-Nan Chen and Li-Ming Tseng \\ Department of Computer Science and Information Engineering, National Central University, Chung-Li 32054, Taiwan \\ Correspondence should be addressed to Ying-Nan Chen, ynchen@dslab.csie.ncu.edu.tw
}

Received 31 January 2012; Accepted 3 February 2012

Academic Editor: Hsiang-Fu Yu

Copyright ( $) 2012$ Y.-N. Chen and L.-M. Tseng. This is an open access article distributed under the Creative Commons Attribution License, which permits unrestricted use, distribution, and reproduction in any medium, provided the original work is properly cited.

\begin{abstract}
Broadcasting Protocols can efficiently transmit videos that simultaneously shared by clients with partitioning the videos into segments. Many studies focus on decreasing clients' waiting time, such as the fixed-delay pagoda broadcasting (FDPB) and the harmonic broadcasting schemes. However, limited-capability client devices such as PDAs and set-top boxes (STBs) suffer from storing a significant fraction of each video while it is being watched. How to reduce clients' buffer demands is thus an important issue. Related works include the staircase broadcasting (SB), the reverse fast broadcasting (RFB), and the hybrid broadcasting $(\mathrm{HyB})$ schemes. This work improves FDPB to save client buffering space as well as waiting time. In comparison with SB, RFB, and $\mathrm{HyB}$, the improved FDPB scheme can yield the smallest waiting time under the same buffer requirements.
\end{abstract}

\section{Introduction}

How to efficiently maintain the exhausted bandwidth with the growth in the number of clients is an important issue of VOD deployment. Dan et al. [1] presented that 80 percent of the demand is for a few number (10 or 20) of very popular videos. One way to broadcast a popular video is to partition the video into segments, which are transmitted on several channels currently and periodically. The approach (called periodic broadcasting [2]) lets multiple users share channels and thus obtains high bandwidth utilization. One of the channels only broadcasts the first segment in real time. The other channels transmit the remaining segments. When clients want to watch a video, they wait for the beginning of the first segment on the first channel. While clients start watching the video, their set-top boxes (STBs) or computers still download and buffer unreceived segments from the channels to enable them to play the video continuously.

The staggered broadcasting [3] scheme treats a complete video as a single segment and then transmits it on each channel at different start times. The fast broadcasting (FB) scheme [4] improves segment partitioning and arrangement to yield shorter service latency. The harmonic broadcasting
(HB) scheme [5] initially partitions a video into equally sized segments, which are further divided into smaller subsegments according to the harmonic series. HB can yield the minimum waiting time [6]; however, its implementation is difficult due to the multitude of broadcasting channels [7]. The recursive frequency-splitting (RFS) [7] scheme broadcasts a segment as close to its frequency as possible to achieve a near-minimal waiting time. The study [8] focuses on reducing the computation complexity of RFS. Paris [9] proposed a fixed-delay pagoda broadcasting (FDPB) scheme that required clients to wait for a small-fixed delay before watching the selected videos.

The staircase broadcasting (SB) scheme [10] requires a client to buffer only $25 \%$ of a playing video. In modifying the FB scheme, the reverse fast broadcasting (RFB) scheme [11] also buffers $25 \%$ of video size, merely half of what is required by the FB scheme. By combining RFS and RFB the hybrid broadcasting scheme (HyB) [12] yields small client buffering space and waiting time. The study in [13] proposed a generalized reverse sequence-based model to reduce their client buffer requirements. This work aims at improving FDPB to reduce required playback latency and buffering space. We prove the applicability of the improved FDPB, and compare 


\begin{tabular}{|c|c|c|}
\hline \multicolumn{3}{|c|}{ Stream 1 } \\
\hline Substream & 1 & 2 \\
\hline First segment & 1 & 2 \\
\hline Last segment & 1 & 3 \\
\hline
\end{tabular}

\begin{tabular}{|c|c|c|}
\hline \multicolumn{3}{|c|}{ Stream 2 } \\
\hline Substream & 1 & 2 \\
\hline First segment & 4 & 7 \\
\hline Last segment & 6 & 10 \\
\hline
\end{tabular}

\begin{tabular}{|c|c|c|c|c|}
\hline \multicolumn{7}{|c|}{ Stream 3 } \\
\hline Substream & 1 & 2 & 3 & 4 \\
\hline First segment & 11 & 14 & 18 & 23 \\
\hline Last segment & 13 & 17 & 22 & 28 \\
\hline
\end{tabular}

(a) The segment allocation on three channels

\begin{tabular}{|l|l|l|l|l|l|l|l|l|l|l|l|l|l|l|l|l|l|l|l|l|l|l|l|l|l|l|l|}
\hline \multicolumn{11}{|c}{ Video length $L$} & $\longrightarrow$ \\
\hline$S_{1}$ & $S_{2}$ & $S_{3}$ & $S_{4}$ & $S_{5}$ & $S_{6}$ & $S_{7}$ & $S_{8}$ & $S_{9}$ & $S_{10}$ & $S_{11}$ & $S_{12}$ & $S_{13}$ & $S_{14}$ & $S_{15}$ & $S_{16}$ & $S_{17}$ & $S_{18}$ & $S_{19}$ & $S_{20}$ & $S_{21}$ & $S_{22}$ & $S_{23}$ & $S_{24}$ & $S_{25}$ & $S_{26}$ & $S_{27}$ & $S_{28}$ \\
\hline
\end{tabular}

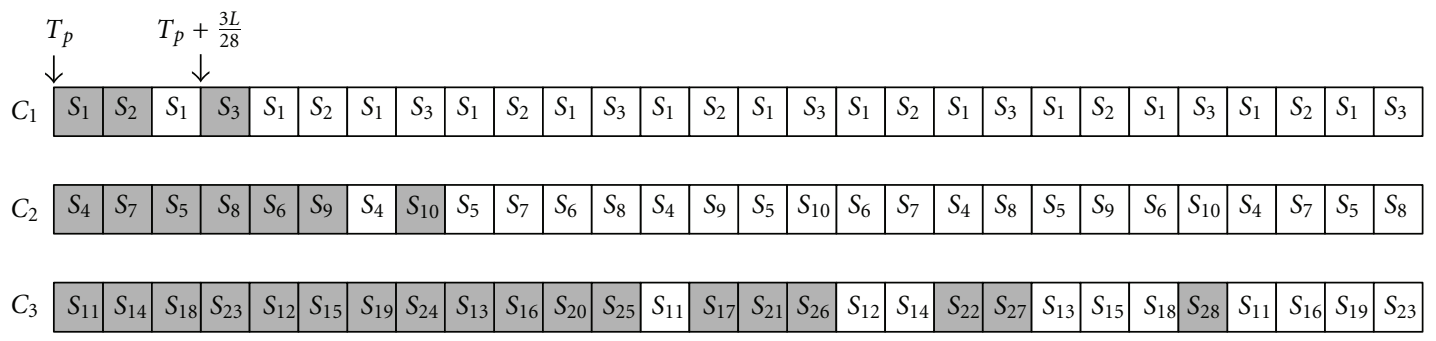

(b) The segment broadcasting on three channels

FIGURE 1: Illustration of channel allocation for the FDPB-3 scheme.

its performance with that of several existing approaches. Given a bandwidth of 5 channels, the new scheme reduces the broadcast latency by as much as $24 \%$ when compared to $\mathrm{HyB}$ and $34.5 \%$ when compared to RFB and SB. The buffer requirements for these schemes are about $25 \%$ of video size.

The rest of this paper is organized as follows. Section 2 introduces FDPB and proposes its improvement. Performance analysis and comparison are in Section 3. We make brief conclusions in Section 4.

\section{Proposed Scheme}

This section briefly reviews FDPB and then presents the improvement.

2.1. Fixed-Delay Pagoda Broadcasting (FDPB) Scheme. To help explain the improved FDPB, we first review the FDPB scheme in the literature [9]. Suppose that a video of duration $L$ is broadcast over $k$ channels. The scheme partitions each video into $m$ equal-size segments of duration $l=L / m$. These segments will be broadcast at different frequencies over the $k$ channels. The FDPB-p scheme requires customers to wait for a fixed time interval $w=p l$, where $p \in$ int, $p \geq 1$. Segment $S_{i}$ is thus transmitted at least once every $p+i-1$ slots. The FDPB-p scheme partitions each channel $C_{j}$ into $c_{j}=\sqrt{p+i-1}$ subchannels in such a way that slot $j$ of channel $C_{j}$ belongs to its subchannel $j$. FDPB then maps segments into subchannels in a strict sequential fashion.

Figure 1 illustrates the video partition and segment arrangement of FDPB-3. Channel $C_{1}$ is partitioned into two subchannels because $\sqrt{3+1-1}$ is close to 2 . Let $S_{i}$ be the $i$ th segment. Since $p=3$, segment $S_{1}$ needs to be broadcast at least once every three slots and is assigned to subchannel 1. The first segment of subchannel 2 is segment $S_{2}$, which appears at least once every $3+2-1=4$ slots. We thus map two segments into subchannel 2, and channel $C_{1}$ transmits three segments (i.e., $S_{1}$ to $S_{3}$ ). The first segment of channel $C_{2}$ is segment $S_{4}$, which is broadcast at least once every $3+4-1=6$ slots. Since 6 is close to $4=2^{2}$, channel $C_{2}$ is partitioned into two subchannels. The first subchannel periodically transmits segments $S_{4}$ to $S_{6}$, and the second subchannel broadcasts segments $S_{7}$ to $S_{10}$. The first segment of channel $C_{3}$ is segment $S_{11}$, which is broadcast at least once every $3+11-1=13$ slots. Similarly, since 13 approaches to $16=4^{2}$, channel $C_{3}$ is partitioned into four subchannels. The first subchannel sends segments $S_{11}$ to $S_{13}$, the second subchannel sends segments $S_{14}$ to $S_{17}$, the third subchannel sends segments $S_{18}$ to $S_{22}$, and the fourth subchannel sends $S_{23}$ to $S_{28}$. Hence, channel $C_{3}$ broadcasts 18 segments. Figure 1(b) demonstrates how to broadcast the segments on three channels and how to download the segments colored gray. Suppose that a client starts receiving the segments at time $T_{p}$. This figure shows that the client begins to watch the video at time $T_{p}+3 L / 28$. 


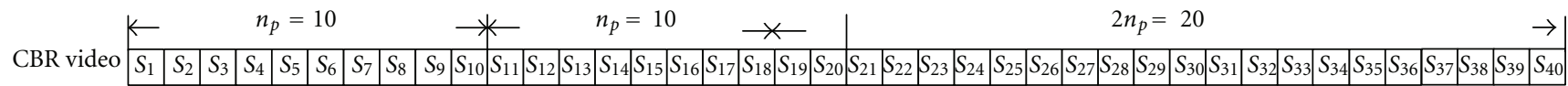

\begin{tabular}{ll|l|l|l|l|l|l|l|l|l|l|l|l|l|l|l|l|l|l|l|l|l|l|l|l|l|l|l|l|l|l|l|l|l|l|l|l}
$C_{1}$ & $S_{1}$ & $S_{2}$ & $S_{1}$ & $S_{3}$ & $S_{1}$ & $S_{2}$ & $S_{1}$ & $S_{3}$ & $S_{1}$ & $S_{2}$ & $S_{1}$ & $S_{3}$ & $S_{1}$ & $S_{2}$ & $S_{1}$ & $S_{3}$ & $S_{1}$ & $S_{2}$ & $S_{1}$ & $S_{3}$ & $S_{1}$ & $S_{2}$ & $S_{1}$ & $S_{3}$ & $S_{1}$ & $S_{2}$ & $S_{1}$ & $S_{3}$ & $S_{1}$ & $S_{2}$ & $S_{1}$ & $S_{3}$ & $S_{1}$ & $S_{2}$ & $S_{1}$ & $S_{3}$ & $\cdots$ \\
\hline
\end{tabular}

\begin{tabular}{ll|l|l|l|l|l|l|l|l|l|l|l|l|l|l|l|l|l|l|l|l|l|l|l|l|l|l|l|l|l|l|l|l|l|l|l|l}
$C_{2}$ & $S_{4}$ & $S_{7}$ & $S_{5}$ & $S_{8}$ & $S_{6}$ & $S_{9}$ & $S_{4}$ & $S_{10}$ & $S_{5}$ & $S_{7}$ & $S_{6}$ & $S_{8}$ & $S_{4}$ & $S_{9}$ & $S_{5}$ & $S_{10}$ & $S_{6}$ & $S_{7}$ & $S_{4}$ & $S_{8}$ & $S_{5}$ & $S_{9}$ & $S_{6}$ & $S_{10}$ & $S_{4}$ & $S_{7}$ & $S_{5}$ & $S_{8}$ & $S_{6}$ & $S_{9}$ & $S_{4}$ & $S_{10}$ & $S_{5}$ & $S_{7}$ & $S_{6}$ & $S_{8}$ & $\ldots$ \\
\hline
\end{tabular}

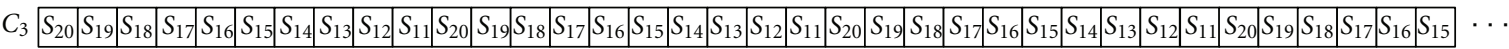

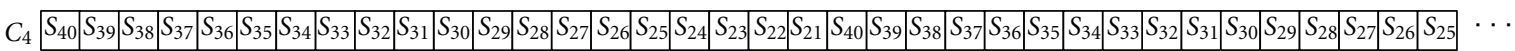

An example, where $k=4$ and $n_{p}=10$

FIGURE 2: Segment transmission by RFDPB-3.

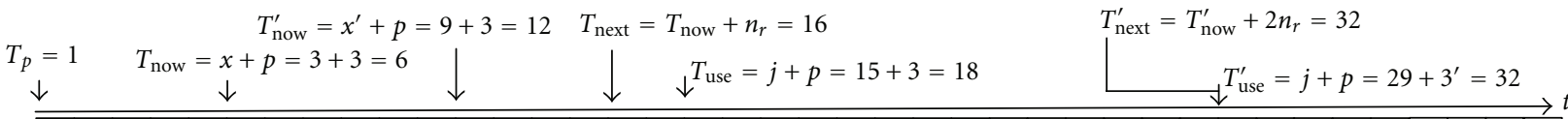

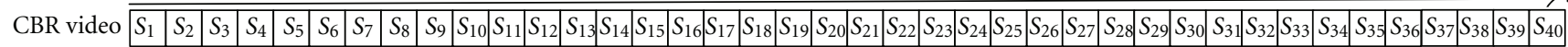

\begin{tabular}{ll|l|l|l|l|l|l|l|l|l|l|l|l|l|l|l|l|l|l|l|l|l|l|l|l|l|l|l|l|l|l|l|l|l|l|l|l}
$C_{1}$ & $S_{1}$ & $S_{2}$ & $S_{1}$ & $S_{3}$ & $S_{1}$ & $S_{2}$ & $S_{1}$ & $S_{3}$ & $S_{1}$ & $S_{2}$ & $S_{1}$ & $S_{3}$ & $S_{1}$ & $S_{2}$ & $S_{1}$ & $S_{3}$ & $S_{1}$ & $S_{2}$ & $S_{1}$ & $S_{3}$ & $S_{1}$ & $S_{2}$ & $S_{1}$ & $S_{3}$ & $S_{1}$ & $S_{2}$ & $S_{1}$ & $S_{3}$ & $S_{1}$ & $S_{2}$ & $S_{1}$ & $S_{3}$ & $S_{1}$ & $S_{2}$ & $S_{1}$ & $S_{3}$ & $\ldots$ \\
\hline
\end{tabular}

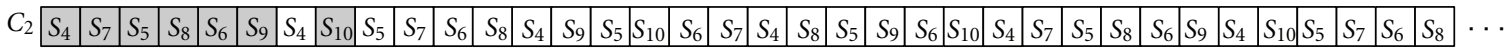

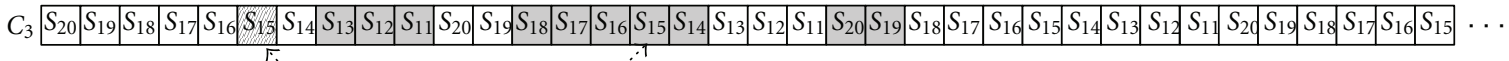

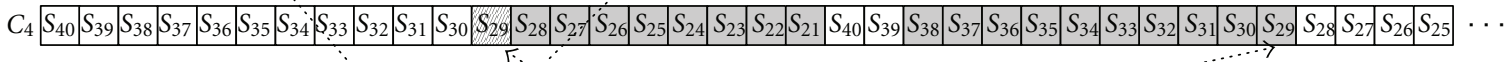

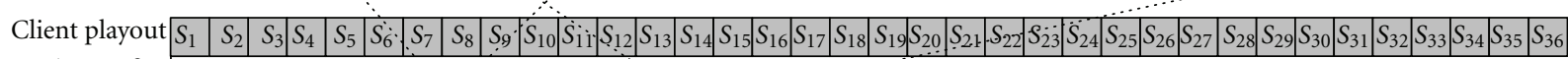

$k P=3 \rightarrow$

$\because S_{x}$

$\because S_{j}{ }^{\top}{ }^{\top} S_{x^{\prime}}$

$S_{j^{\prime}}$

FIgURE 3: An illustration of segment downloading by RFDPB-3, where $k=4, n_{p}=10$, and $N=40$.

2.2. Improvement of FDPB. By integrating the $\mathrm{RFB}$ with FDPB, this work designs the RFB-FDPB (RFDPB) scheme, which gains small low buffer requirements as well as waiting time.

2.2.1. Segment Delivery on the Server Side. Let $k$ be the number of allocated channels. The data transmission rate of each channel is assumed to equal the playback rate $b$. An RFDPB server broadcasts segments according to the following steps.

(1) The largest number of segments that FDPB- $p$ can transmit using $k-2$ channels is assumed to equal $n_{p}$. The RFDPB- $p$ scheme then equally divides a constant-bit-rate $(\mathrm{CBR})$ video into $N$ segments, denoted by $S_{1}, S_{2}, \ldots, S_{N}$ in sequence, where $N=4 n_{p}$. Let $L$ be the length of the video. The length of a segment, $l$, thus equals $L / N$.

(2) Channels $C_{1}$ to $C_{k-2}$ periodically send segments $S_{1}$ to $S_{n_{p}}$ according to FDPB-p.

(3) Segments $S_{2 n_{p}}$ to $S_{n_{p}+1}$ are transmitted on channel $C_{k-1}$.

(4) Segments $S_{4 n_{p}}$ to $S_{2 n_{p}+1}$ are broadcast on channel $C_{k}$.
Figure 2 illustrates the segment delivery by RFDPB-3, where $k=4$. The server broadcasts segments $S_{1}$ to $S_{10}$ on channels $C_{1}$ to $C_{2}$ by FDPB-3 because FDPB-3 can transmit 10 segments using two channels, as indicated in Figure 1. Due to $n_{p}=10$, RFDPB periodically broadcasts segments $S_{20}$ to $S_{11}$ and $S_{40}$ to $S_{21}$ on channels $C_{3}$ and $C_{4}$.

2.2.2. Segment Reception on the Client Side. Suppose that a client has enough buffers to store portions of a playing video. Let $l$ be a basic time unit during video playing. We also let $T_{p}$ be the time that the client starts downloading the video and be the origin (i.e., the first time unit) of the time axis throughout the paper. The following steps are involved in playing a video at the client.

(1) The client receives the segments on channels $C_{1}$ to $C_{k-2}$ immediately when they are available on networks. Figure 3 further demonstrates the segment downloading, where the segments downloaded by a client are gray.

(2) The segment downloading on channel $C_{k-1}$ is as follows. Suppose that the client first sees segment $S_{j}$ on channel $C_{k-1}$ at time $T_{\text {now }}$ and next segment 


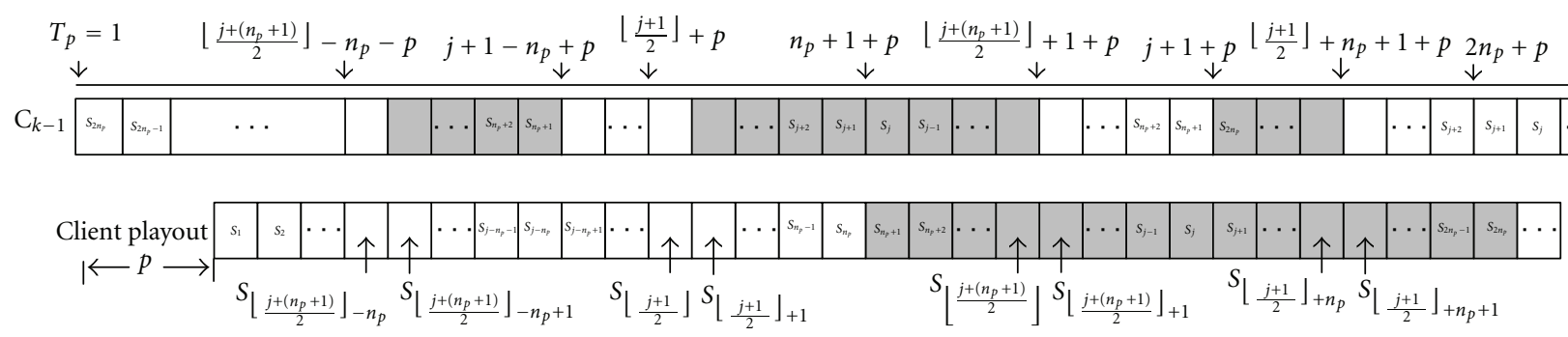

(Not to scale)

Figure 4: The segment downloading on channel $C_{k-1}$ under RFDPB- $p$.
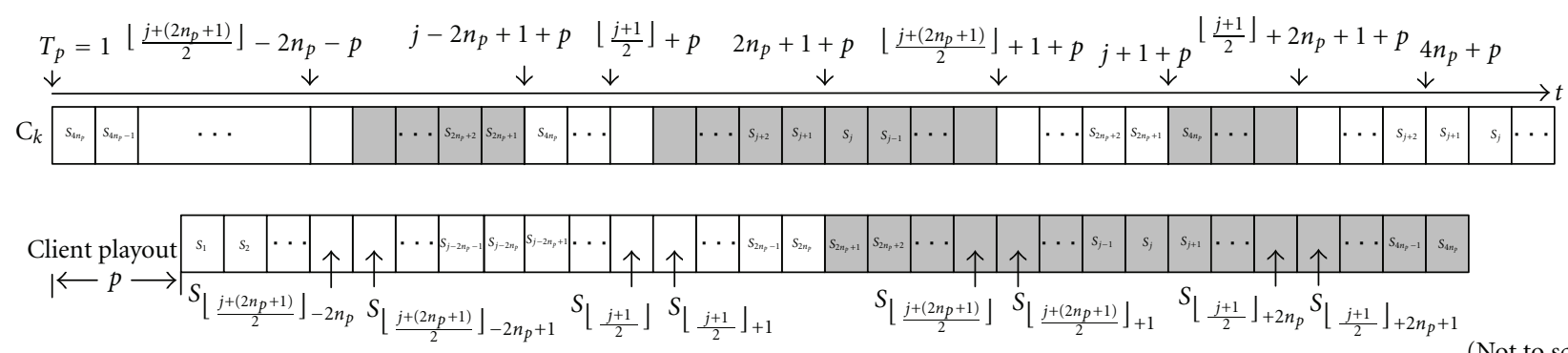

(Not to scale)

Figure 5: The segment downloading on channel $C_{k}$ under RFDPB- $p$.

$S_{j}$ at time $T_{\text {next }}$. The client is also assumed to play segments $S_{x}$ and $S_{j}$ at time $T_{\text {now }}$ and $T_{\text {use }}$, respectively. The work in $[11,12]$ indicates that if $T_{\text {next }} \leq T_{\text {use }}$, the client does not need to receive segment $S_{j}$ at time $T_{\text {now }}$ and actually performs the downloading at time $T_{\text {next }}$, without causing playing interruption. FDPB further reveals that a client has to wait for a fix time unit $p$ to play received segments. Thus, $T_{\text {use }}=j+p$ th time unit, $T_{\text {now }}=x+p$ th time unit, and $T_{\text {next }}=T_{\text {now }}+n_{p}$. Substituting these equations into $T_{\text {next }} \leq T_{\text {use }}$, we obtain

$$
x+n_{p} \leq j .
$$

If this inequality holds, a client does not receive segment $S_{j}$ at time $T_{\text {now }}$; otherwise, it immediately downloads it. Suppose that the client first sees segment $S_{15}$ with only diagonal lines on channel $C_{3}$ at the 6th time unit, as indicated in Figure 3. Due to $p=3, x=3, j=15$, and $n_{p}=10, T_{\text {now }}=x+p=6$, $T_{\text {use }}=j+p=18$, and $T_{\text {next }}=T_{\text {now }}+n_{p}=16$. Clearly, it is unnecessary to download segment $S_{15}$ at this time unit because inequality (1), $x+n_{p}=13 \leq j=15$, holds. The result also reflects that the client does not have to receive the segment when $T_{\text {next }}=16 \leq T_{\text {use }}=$ 18. When next segment $S_{15}$ colored gray in Figure 3 arrives at the 16th time unit, the inequality is no more true, $13+10>15$, and the client thus downloads it.

(3) The segments on channel $C_{k}$ are received in the similar way, as also indicated in Figure 3. Suppose that the client first sees segment $S_{j^{\prime}}$ on channel $C_{k}$ at time $T^{\prime}{ }_{\text {now }}$, and next segment $S_{j^{\prime}}$ at time $T^{\prime}$ next. The client is also assumed to play segments $S_{x^{\prime}}$ and $S_{j^{\prime}}$ at time $T^{\prime}$ now and $T^{\prime}$ use. Similarly, if $T^{\prime}{ }_{\text {next }} \leq T^{\prime}$ use, the client does not receive segment $S_{j^{\prime}}$. Substituting $T^{\prime}{ }_{\text {use }}=j^{\prime}+p$ th time unit, $T_{\text {now }}^{\prime}=x^{\prime}+p$ th time unit, and $T^{\prime}{ }_{\text {next }}=T^{\prime}{ }_{\text {now }}+2 n_{p}$ into inequality $T^{\prime}{ }_{\text {next }} \leq T^{\prime}$ use obtains

$$
x^{\prime}+2 n_{p} \leq j^{\prime} .
$$

If the inequality is true, the client skips the downloading of segment $S_{j^{\prime}}$. Suppose that the client first sees segment $S_{29}$ with only diagonal lines on channel $C_{4}$ at the 12th time unit, as indicated in Figure 3. Due to $p=3, x^{\prime}=9, j^{\prime}=29$, and $n_{p}=10, T_{\text {now }}^{\prime}=x^{\prime}+p=$ $12, T_{\text {use }}^{\prime}=j^{\prime}+p=32$, and $T_{\text {next }}^{\prime}=T_{\text {now }}^{\prime}+2 n_{p}=32$. Clearly, it is unnecessary to download segment $S_{29}$ at this time unit because inequality (2), $x^{\prime}+2 n_{p}=$ $29 \leq j^{\prime}=29$, is true. The result also reflects that the client does not have to receive the segment when $T_{\text {next }}^{\prime}=32 \leq T_{\text {use }}^{\prime}=32$. When next segment $S_{29}$ colored gray in Figure 3 arrives at the 32 nd time unit, the inequality is no more true, $29+20>29$, and the client thus downloads it.

(4) The client plays the video in the order of $S_{1}, S_{2}, \ldots, S_{N}$ at time $T_{p}+p$.

(5) Stop loading data from networks when all the segments have been received. 

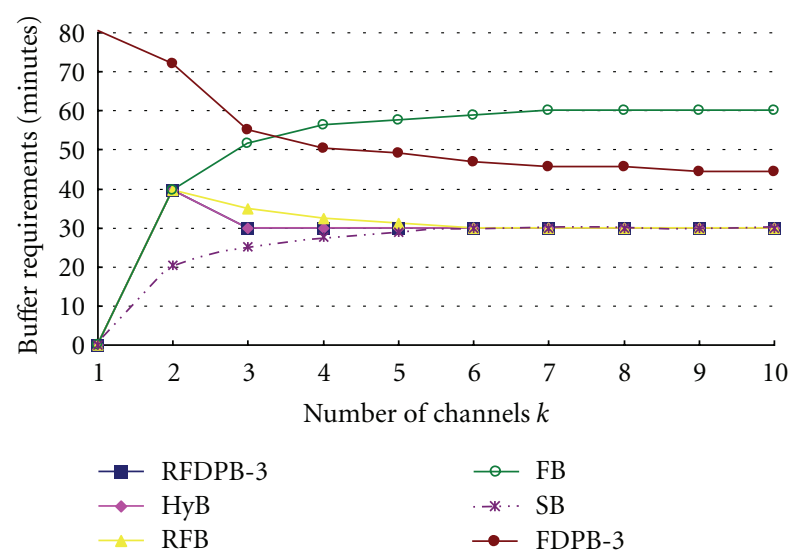

Figure 6: Comparison of the maximum buffer requirements in the number of minutes of a 120-minute video.

TABLE 1: The maximum buffering space required by different schemes in the percentage of video size using $k$ channels.

\begin{tabular}{lcccccccccc}
\hline$k$ & 1 & 2 & 3 & 4 & 5 & 6 & 7 & 8 & 9 & 10 \\
\hline RFDPB-3 & 0 & 33 & 25 & 25 & 25 & 25 & 25 & 25 & 25 & 25 \\
HyB & 0 & 33 & 25 & 25 & 25 & 25 & 25 & 25 & 25 & 25 \\
RFB & 0 & 33 & 29 & 27 & 26 & 25 & 25 & 25 & 25 & 25 \\
FB & 0 & 33 & 43 & 47 & 48 & 49 & 50 & 50 & 50 & 50 \\
SB & 0 & 17 & 21 & 23 & 24 & 25 & 25 & 25 & 25 & 25 \\
FDPB-3 & 67 & 60 & 46 & 42 & 41 & 39 & 38 & 38 & 37 & 37 \\
\hline
\end{tabular}

2.2.3. Workable Verification. This section describes that the RFDPB scheme guarantees continuous playing on the client side. Because the segment broadcasting on channels $C_{1}$ to $C_{k-2}$ is based on FDPB [9], RFDPB is workable for these channels. We next prove that the segment arrangements on channels $C_{k-1}$ and $C_{k}$ also ensure a client to continuously play a video. FDPB further indicates that a video server must broadcast segment $S_{j}$ at least once in every $j+p-1$ time units to keep on-time data delivery on the client side. For the RFDPB scheme, a server broadcasts segment $S_{j}$ on channel $C_{k-1}$ in every $n_{p}$ time units, where $n_{p}+1 \leq j \leq 2 n_{p}$. Because $j+p-1>n_{p}$, an RFDPB client can receive video segments on channel $C_{k-1}$ on time. Similarly, the RFDPB scheme requires a server to transmit segment $S_{j^{\prime}}$ on channel $C_{k}$ in every $2 n_{p}$ time units, where $2 n_{p}+1 \leq j^{\prime} \leq 4 n_{p}$. Because $j^{\prime}+p-1>2 n_{p}$, the segment delivery on this channel is also on time. Accordingly, RFDPB ensures continuous video playing at the client.

\section{Analysis and Comparison}

Before investigating entire buffer requirements for RFDPB, this paper analyzes the segment downloading on channel $C_{k-1}$ first. For RFDPB- $p$, a client plays the segments received from this channel during time units $n_{p}+1+p$ to $2 n_{p}+p$. The possible time to download the segments is during the $p$ th to $2 n_{p}+p$ th time units. Suppose that a client sees segment $S_{j}$ at the $n_{p}+1+p$ th time unit. Inequality (1) makes a
TABLE 2: The maximum numbers of segments, $N$, offered by different schemes.

\begin{tabular}{lcccccccccc}
\hline$k$ & 1 & 2 & 3 & 4 & 5 & 6 & 7 & 8 & 9 & 10 \\
\hline FDPB-6 & 7 & 25 & 71 & 186 & 485 & 1286 & 3425 & 9195 & 24790 & 67054 \\
RFDPB-6 & 1 & 3 & 28 & 100 & 284 & 744 & 1940 & 5144 & 13700 & 36780 \\
HyB & 1 & 3 & 4 & 12 & 36 & 100 & 292 & 804 & 2260 & 6088 \\
FB (RFB) & 1 & 3 & 7 & 15 & 31 & 63 & 127 & 255 & 511 & 1023 \\
SB & 1 & 3 & 7 & 15 & 31 & 63 & 127 & 255 & 511 & 1023 \\
\hline
\end{tabular}

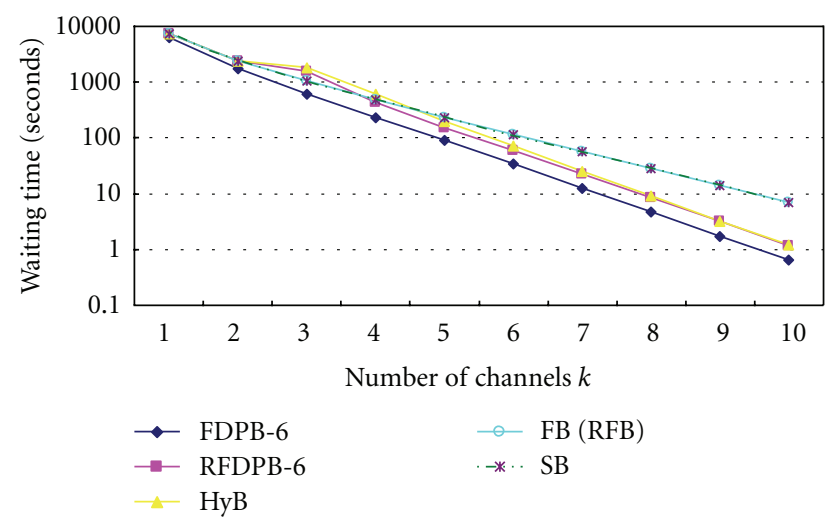

FIGURE 7: The maximum waiting time incurred on new clients at different numbers of channels ( $L=120$ minutes).

complete segment-downloading diagram for channel $C_{k-1}$, as indicated in Figure 4, where the segments downloaded by the client are colored gray. The figure shows that the client does not continuously download segments, and some segments are skipped. The segments are not downloaded during the first to $\left\lfloor\left(j+\left(n_{p}+1\right)\right) / 2\right\rfloor-n_{p}+p$ th time units, during the $j+1-n_{p}+p$ th to $\lfloor(j+1) / 2\rfloor+p$ th time units, during the $\left\lfloor\left(j+\left(n_{p}+1\right)\right) / 2\right\rfloor+1+p$ th to $j+p$ th time units, and during the $\lfloor(j+1) / 2\rfloor+n_{p}+1+p$ th to $2 n_{p}+p$ th time units, respectively. Such results reflect that the client can delay downloading these segments by $n_{p}$ time units, due to (1). Similarly, (2) also makes a complete segment-downloading diagram for channel $C_{k}$, as indicated in Figure 6, where a client is assumed to see segment $S_{j}$ at the $2 n_{p}+1+p$ th time unit.

The RFDPB scheme uses the same methodology as HyB, combining a nonharmonic scheme and the RFB to reduce clients' waiting time and buffering spaces (Figure 5). For segment broadcasting on channels $C_{1}$ to $C_{k-2}$ using the nonharmonic scheme, channels $C_{k-1}$ and $C_{k}$ broadcast the other segments according RFB. Yu's work [12] has proved HyB only buffer $25 \%$ of video size for $k \geq 3$, where $k$ is the number of broadcasting channels. The proof of the maximum number of segments buffered by a client for RFDPB is similar to that of HyB, so this paper neglects it. Therefore, let $B(k)$ be the maximum number of segments buffered by a client, where $k$ is the number of broadcasting channels. For RFDPB,

$$
\begin{gathered}
B(1)=0 \\
B(2)=1 \\
B(k)=n_{p}, \quad k \geq 3 .
\end{gathered}
$$


TABLE 3: The waiting time (seconds), offered by RFDPB-9, RFDPB-6, HyB, and RFDPB-3 schemes.

\begin{tabular}{lcccccccccc}
\hline$k$ & 1 & 2 & 3 & 4 & 5 & 6 & 7 & 8 & 9 \\
\hline RFDPB-9 & 7200 & 2400 & 1350 & 385.71 & 139.66 & 52.6 & 19.9 & 7.47 & 2.79 & 1.04 \\
RFDPB-6 & 7200 & 2400 & 1542.86 & 432 & 152.11 & 58.06 & 22.27 & 8.4 & 3.15 & 1.17 \\
HyB & 7200 & 2400 & 1800 & 600 & 200 & 72 & 24.66 & 8.96 & 3.19 & 1.18 \\
RFDPB-3 & 7200 & 2400 & 1800 & 540 & 192.86 & 72.97 & 28.57 & 11.07 & 4.19 & 1.58 \\
\hline
\end{tabular}

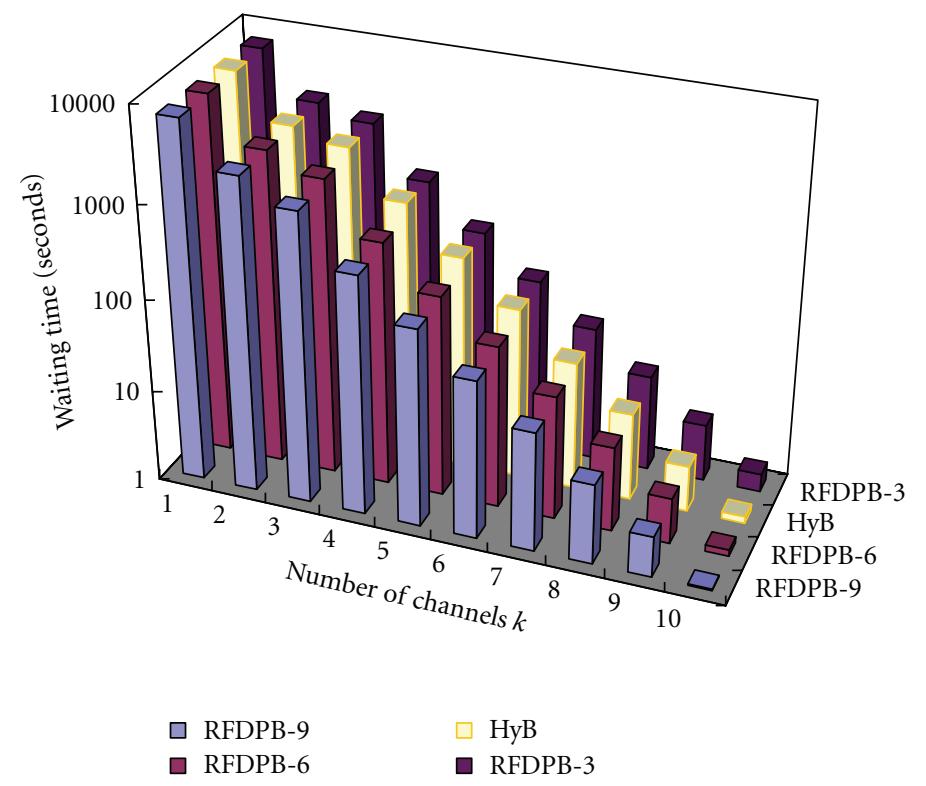

FIGURE 8: The maximum waiting time (RFDPB and HyB) incurred on new clients at different numbers of channels $(L=120$ minutes).

The previous analysis indicates that RFDPB buffers at most $n_{r} / N=25 \%$ of video size. Table 1 lists the comparison of maximum buffering spaces required by RFDPB-3, FB, SB, RFB, FDPB-3, and HyB. RFDPB-3 reduces the buffering space up to $32.4 \%$ when compared to FDPB-3 and up to $50 \%$ when compared to $\mathrm{FB}$ and has the same good result with the other schemes. Given a video of 120 minutes, Figure 6 shows the buffer requirements at different channels. To understand how well the RFDPB scheme performs on clients waiting time, this work calculates the values of $N$ offered by RFDPB6, SB, FB, RFB, FDPB-6, and HyB given different numbers of server channels, as listed in Table 2. The inverse of $N$ offered by each scheme reflects the waiting time for a new client to start his/her VOD service. Figure 7, which is drawn in a logarithmic scale, shows that RFDPB-6 performs close to FDPB-6 stably. For $k \geq 4$, the RFDPB-6 outperforms all the schemes, except the FDPB-6. To understand the relationship between RFDPB and HyB schemes, this work calculates clients' waiting time of RFDPB-9, RFDPB-6, $\mathrm{HyB}$, and RFDPB-3, as listed in Table 3. Figure 8 shows that, for $p \geq 6$, RFDPB-p outperforms HyB. The previous comparisons clearly indicate that RFDPB exhibits a good tradeoff between client buffering spaces and waiting time.

\section{Conclusions}

This paper presents an improved version of FDPB for efficient periodic broadcasting of popular videos. The proposed scheme takes advantage of the FDPB and the RFB schemes to obtain small waiting time and low buffer demand. Through mathematical analysis, we prove the applicability of this scheme by demonstrating that client playback continuity is guaranteed. Given a bandwidth of 5 channels, the new scheme reduces the broadcast latency by as much as $24 \%$ when compared to $\mathrm{HyB}$ and $34.5 \%$ when compared to RFB and SB. The buffer requirements for these schemes are about $25 \%$ of video size.

\section{References}

[1] A. Dan, D. Sitaram, and P. Shahabuddin, "Dynamic batching policies for an on-demand video server," Multimedia Systems, vol. 4, no. 3, pp. 112-121, 1996.

[2] D. Saparilla, K. W. Ross, and M. Reisslein, "Periodic broadcasting with VBR-encoded video," in Proceedings of the 18th Annual Joint Conference of the IEEE Computer and Communications Societie (INFOCOM '99), pp. 464-471, March 1999.

[3] K. C. Almeroth and M. H. Ammar, "The use of multicast delivery to provide a scalable and interactive video-on-demand service," IEEE Journal on Selected Areas in Communications, vol. 14, no. 6, pp. 1110-1122, 1996.

[4] L-S. Juhn and L. M. Tseng, "Fast data broadcasting and receiving scheme for popular video service," IEEE Transactions on Broadcasting, vol. 44, no. 1, pp. 100-105, 1998.

[5] L.-S. Juhn and L. M. Tseng, "Harmonic broadcasting for video-on-demand service," IEEE Transactions on Broadcasting, vol. 43, no. 3, pp. 268-271, 1997. 
[6] Z.-Y. Yang, L. S. Juhn, and L. M. Tseng, "On optimal broadcasting scheme for popular video service," IEEE Transactions on Broadcasting, vol. 45, no. 3, pp. 318-322, 1999.

[7] Y.-C. Tseng, M. H. Yang, and C. H. Chang, "A recursive frequency-splitting scheme for broadcasting hot videos in VOD service," IEEE Transactions on Communications, vol. 50, no. 8 , pp. 1348-1355, 2002.

[8] J.-P. Sheu, H. L. Wang, C. H. Chang, and Y. C. Tseng, "A fast video-on-demand broadcasting scheme for popular videos," IEEE Transactions on Broadcasting, vol. 50, no. 2, pp. 120-125, 2004.

[9] J. F. Paris, "A fixed-delay broadcasting protocol for video-ondemand," in Proceedings of the 10th International Conference on Computer Communications and Networks (ICCCN'01), pp. 418-423, Scottsdale, Ariz, USA, October 2001.

[10] L.-S. Juhn and L. M. Tseng, "Staircase data broadcasting and receiving scheme for hot video service," IEEE Transactions on Consumer Electronics, vol. 43, no. 4, pp. 1110-1117, 1997.

[11] H.-F. Yu, H. C. Yang, and L. M. Tseng, "Reverse Fast Broadcasting (RFB) for video-on-demand applications," IEEE Transactions on Broadcasting, vol. 53, no. 1, pp. 103-110, 2007.

[12] H.-F. Yu, "Hybrid broadcasting with small buffer demand and waiting time for video-on-demand applications," IEEE Transactions on Broadcasting, vol. 54, no. 2, Article ID 4433980, pp. 304-311, 2008.

[13] H.-F. Yu, P. H. Ho, and H. C. Yang, "Generalized SequenceBased and Reverse Sequence-Based Models for Broadcasting Hot Videos," IEEE Transactions on Multimedia, 2008. 

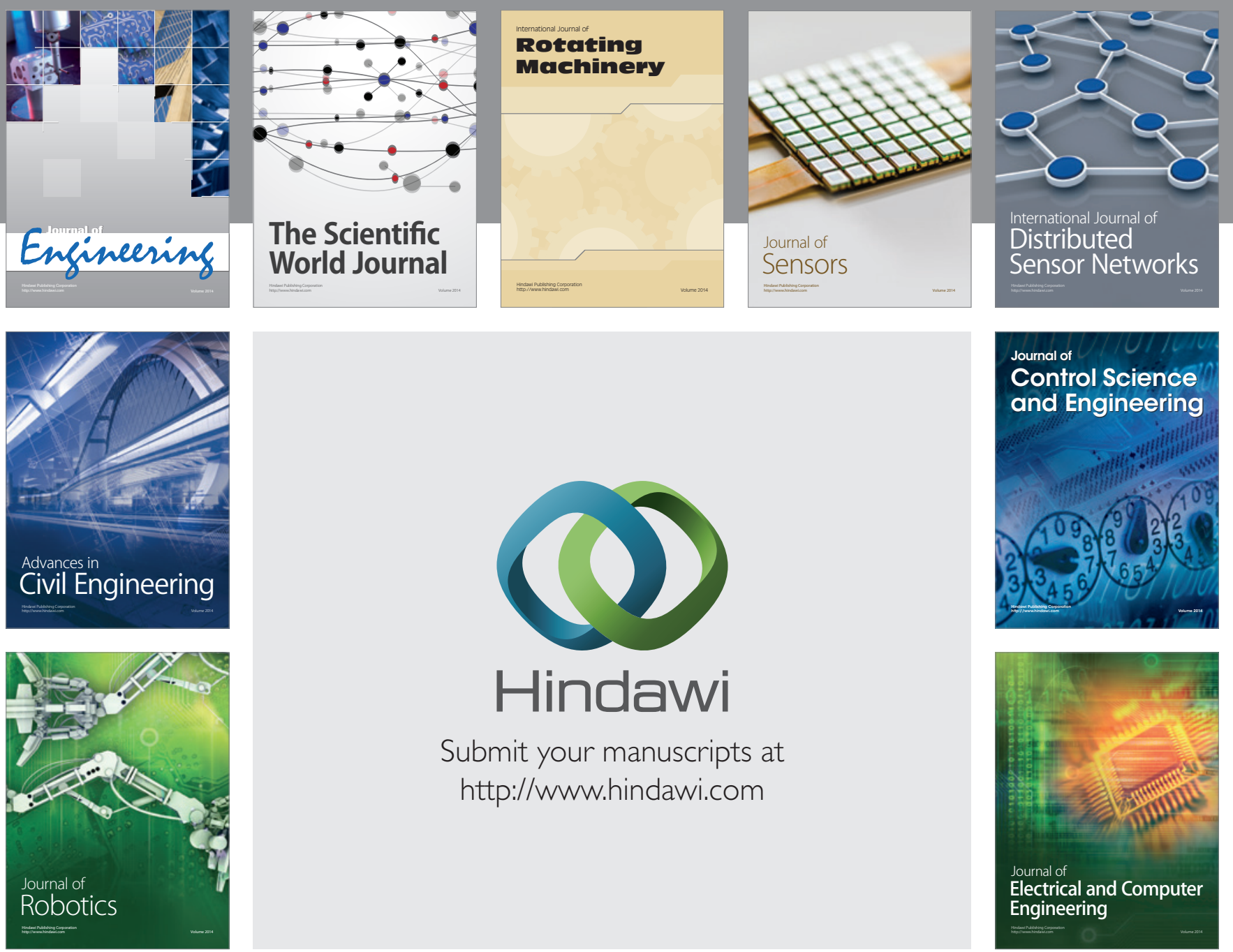

Submit your manuscripts at

http://www.hindawi.com
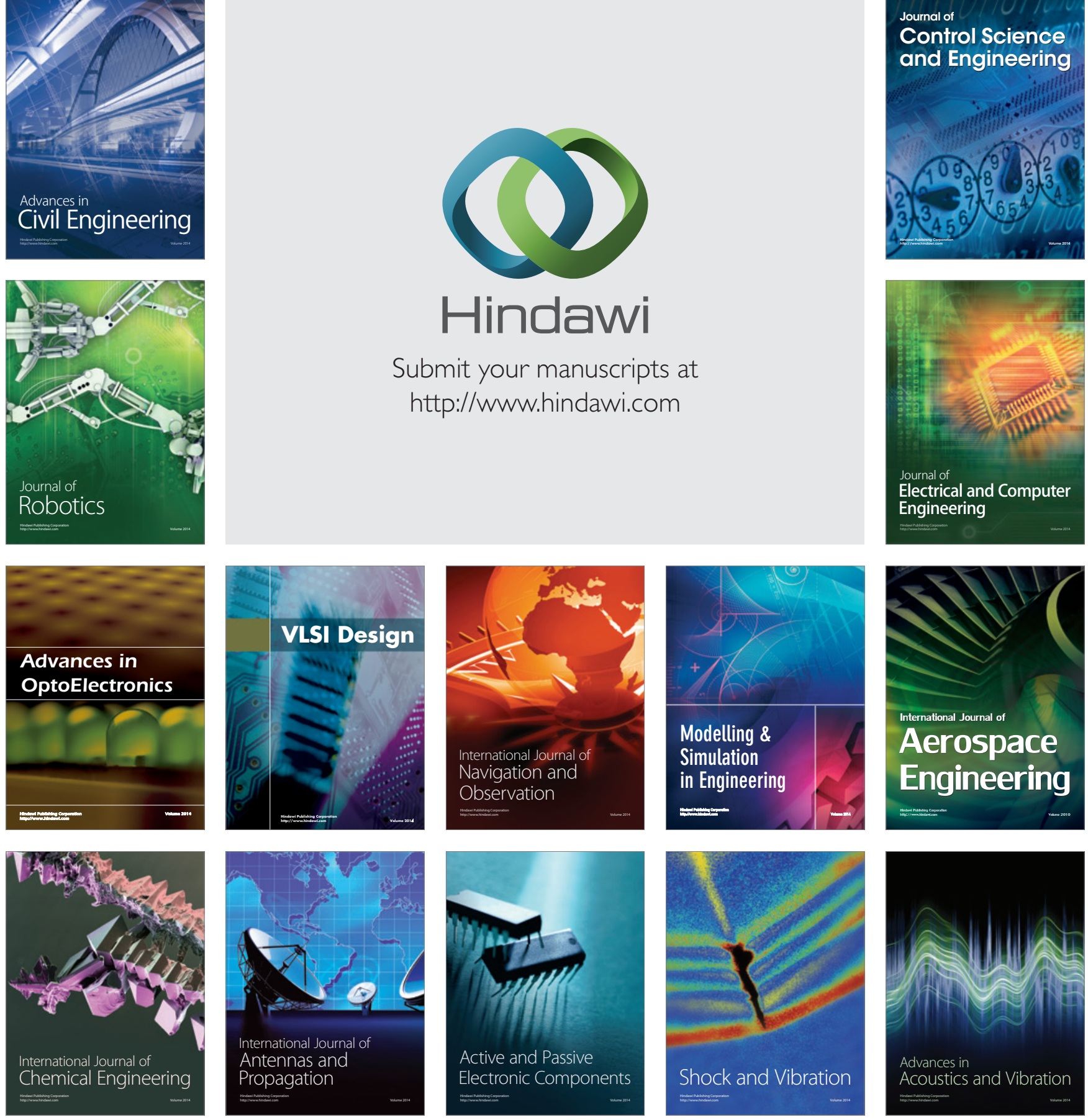